DRAFt VERSION OCTOBER 23, 2020

Typeset using LATEX RNAAS style in AASTeX63

\title{
Preliminary Target Selection for the DESI Luminous Red Galaxy (LRG) Sample
}

\author{
Rongpu Zhou, ${ }^{1,2}$ Jeffrey A. Newman, ${ }^{2}$ Kyle S. Dawson, ${ }^{3}$ Daniel J. Eisenstein, ${ }^{4}$ David D. Brooks, ${ }^{5}$ Arjun Dey, ${ }^{6}$ \\ Biprateep Dey, ${ }^{2}$ Yutong Duan, ${ }^{7}$ Sarah Eftekharzadeh, ${ }^{8}$ Enrique Gaztañaga, ${ }^{9,}{ }^{10}$ Robert Kehoe, ${ }^{11}$ Martin Landriau, ${ }^{1}$ \\ Michael E. Levi, ${ }^{1}$ Timothy C. Licquia, ${ }^{2}{ }^{12}$ Aaron M. Meisner, ${ }^{6}$ John Moustakas, ${ }^{13}$ Adam D. Myers, ${ }^{14}$ \\ Nathalie Palanque-Delabrouille, ${ }^{15}$ Claire Poppett,${ }^{16}$ Francisco Prada, ${ }^{17}$ Anand Raichoor, ${ }^{18}$ David J. Schlegel, ${ }^{1}$ \\ Michael Schubnell, ${ }^{19}$ Ryan Staten, ${ }^{11}$ Gregory Tarlé, ${ }^{20}$ and Christophe Yèche ${ }^{15}$ \\ ${ }^{1}$ Lawrence Berkeley National Laboratory, 1 Cyclotron Road, Berkeley, CA 94720, USA \\ ${ }^{2}$ University of Pittsburgh, 100 Allen Hall, 3941 O'Hara St., Pittsburgh, PA 15260, USA \\ 3 Department of Physics and Astronomy, University of Utah, Salt Lake City, UT 84112, USA \\ ${ }^{4}$ Harvard-Smithsonian Center for Astrophysics, 60 Garden St, Cambridge, MA 02138 \\ ${ }^{5}$ Department of Physics 83 Astronomy, University College London, Gower Street, London, WC1E 6BT, UK \\ ${ }^{6}$ NSF's National Optical-Infrared Astronomy Research Laboratory, 950 N. Cherry Ave., Tucson, AZ 85719, USA \\ ${ }^{7}$ Physics Department, Boston University, Boston, MA 02215, MA \\ ${ }^{8}$ Department of Physics and Astronomy, The University of Utah, 115 South 1400 East, Salt Lake City, UT 84112, USA \\ ${ }^{9}$ Institute of Space Sciences (ICE, CSIC), 08193 Barcelona, Spain \\ 10 Institut d'Estudis Espacials de Catalunya (IEEC), 08034 Barcelona, Spain \\ ${ }^{11}$ Department of Physics, Southern Methodist University, 3215 Daniel Avenue, Dallas, TX 75275, USA \\ ${ }^{12}$ Dow Chemical Company, 2200 W. Salzburg Road, PO Box 994, Auburn, MI 48611 \\ ${ }^{13}$ Department of Physics 8 Astronomy, Siena College, 515 Loudon Road, Loudonville, NY 12211, USA \\ ${ }^{14}$ University of Wyoming, 1000 E. University Ave., Laramie, WY 82071, USA \\ ${ }^{15}$ IRFU, CEA, Université Paris-Saclay, F-91191 Gif-sur-Yvette, France \\ ${ }^{16}$ Space Sciences Laboratory at University of California, 7 Gauss Way, Berkeley, CA 94720 \\ ${ }^{17}$ Instituto de Astrofisica de Andalucía, Glorieta de la Astronomía, s/n, E-18008 Granada, Spain \\ ${ }^{18}$ Institute of Physics, Laboratory of Astrophysics, Ecole Polytechnique Fédérale de Lausanne (EPFL), Observatoire de Sauverny, 1290 \\ Versoix, Switzerland \\ ${ }^{19}$ Department of Physics, University of Michigan, 450 Church St., Ann Arbor, MI 48109, USA \\ ${ }^{20}$ Department of Physics, University of Michigan, Ann Arbor, MI 48109, USA
}

\begin{abstract}
The DESI survey will observe more than 8 million candidate luminous red galaxies (LRGs) in the redshift range $0.3<z<1.0$. Here we present a preliminary version of the DESI LRG target selection developed using Legacy Surveys Data Release $8 g, r, z$ and $W 1$ photometry. This selection yields a sample with a uniform surface density of $\sim 600 \mathrm{deg}^{-2}$ and very low predicted stellar contamination and redshift failure rates. During DESI Survey Validation, updated versions of this selection will be tested and optimized.
\end{abstract}

Keywords: surveys, large-scale structure, cosmology: observations

\section{INTRODUCTION}

The Dark Energy Spectroscopic Instrument (DESI, DESI Collaboration et al. 2016) will be used to obtain spectra of more than 8 million luminous red galaxies (LRGs) in the redshift range $0.3<z<1.0$, in addition to samples of stars, quasars and other galaxies. LRGs are massive galaxies that have typically ceased star formation and which occupy highly biased structures. Their spectra have a strong $4000 \AA$ break, which makes their redshifts relatively easy to measure. These properties make LRGs an ideal tracer for mapping the large-scale structure of the Universe. Here, we describe a preliminary DESI LRG selection designed using Data Release 8 (DR8) of the DESI Legacy Imaging Surveys (Dey et al. 2019) ${ }^{1}$, and make the resulting target catalogs public ${ }^{2}$.

${ }^{1}$ http://legacysurvey.org/dr8/

${ }^{2}$ Available at https://data.desi.lbl.gov/public/ets/target/catalogs/ and detailed at https://desidatamodel.readthedocs.io 


\section{LRG TARGET SELECTION}

Our LRG targets are selected using Legacy Surveys (LS) DECaLS/BASS/MzLS $g, r, z$, and WISE (Wright et al. 2010) $W 1$ photometry (Dey et al. 2019). The targets are much brighter than the limits of the imaging data. As a result the selection is insensitive to variations in imaging properties such as depth, yielding a uniform surface density across the survey footprint.

For lower-redshift LRGs, it is possible to select objects with red optical colors exploiting the $4000 \AA$ break, as done for SDSS-I (Eisenstein et al. 2001) and BOSS (Reid et al. 2016), but such methods fail when the break moves beyond the $r$ band. For the eBOSS survey (Dawson et al. 2016), WISE photometry was used to reject stars and efficiently select massive galaxies at higher redshift (see Prakash et al. 2015, 2016). This method takes advantage of the prominent $1.6 \mu \mathrm{m}$ (restframe) "bump" (John 1988; Sawicki 2002) which produces an excess of flux in the $W 1$ (3.4 $\mu \mathrm{m})$ band for $z \sim 1$ galaxies.

The color and magnitude cuts used to select LRGs in the DECaLS (Southern) DR8 imaging are:

$$
\begin{aligned}
& (z-W 1)>0.8 \times(r-z)-0.6, \\
& ((g-W 1>2.6) \text { AND }(g-r>1.4)) \text { OR }(r-W 1>1.8), \\
& (r-z>(z-16.83) \times 0.45) \text { AND }(r-z>(z-13.80) \times 0.19), \\
& r-z>0.7, \\
& \text { and } z_{\text {fiber }}<21.5,
\end{aligned}
$$

where $g, r, z$, and $W 1$ indicate the extinction-corrected $\mathrm{AB}$ magnitudes in the corresponding band (using LS extinction corrections $^{3}$ ), and $z_{\text {fiber }}$ is the magnitude corresponding to the expected $z$ flux within a DESI fiber. Tweaks are needed to select an equivalent sample in the Northern (BASS/MzLS) DR8 imaging due to differences in instruments and passbands; the cuts which differ are:

$$
\begin{aligned}
& (z-W 1)>0.8 \times(r-z)-0.65, \\
& ((g-W 1>2.67) \text { AND }(g-r>1.45)) \text { OR }(r-W 1>1.85), \\
& \text { and }(r-z>(z-16.69) \times 0.45) \text { AND }(r-z>(z-13.68) \times 0.19) .
\end{aligned}
$$

We require all targets to be covered by at least one image in each optical band. We remove saturated objects and sources near bright stars, large galaxies, or globular clusters by requiring that LS MASKBITS ${ }^{4} 1,5,6,7,11,12$, and 13 are not set.

The boundaries in color-magnitude space corresponding to Equations 1a-e and the predicted redshift distribution for the sample are depicted in Figure 1. The first cut (shown in the upper left panel of Figure 1) provides an effective rejection of stars, much as in Prakash et al. (2016). The cuts shown in the upper-right panel eliminate low-redshift or bluer objects. As shown in the lower-left panel, applying a magnitude limit which is a function of color allows only the most luminous objects at a given redshift to be selected. In addition to these cuts, we also apply a limit on $z_{\text {fiber }}$ to ensure targets yield secure redshift measurements.

We have also developed alternative approaches which apply a single, broader $r-W 1$ cut independent of $g-r$, and which employ a magnitude limit in the $r-W 1$ vs. $W 1$ plane instead of in $z$. The resulting selection eliminates $g$ and reduces $z$-band dependence (making it less sensitive to photometric calibration errors) but still yields a similar redshift distribution to that shown in Figure 1.

\section{CONCLUSIONS}

LRG targets selected as outlined in this Note have a total surface density of $\sim 600 \mathrm{deg}^{-2}$ and a roughly constant comoving density of $\sim 6 \times 10^{-4} h^{3} \mathrm{Mpc}^{-3}$ over the redshift range $0.3<z<0.8$. Tests with DESI commissioning data suggest that in nominal conditions $>98 \%$ of these targets should yield secure redshift measurements, with $<1 \%$ stellar contamination at high Galactic latitudes. Legacy Surveys DR9 imaging and DESI Survey Validation (SV) spectroscopy will soon be used to choose the best method for LRG targeting and optimize the selection cuts for the DESI survey. We expect the DESI LRG sample to meet or exceed all relevant Science Requirements ${ }^{5}$ and to surpass previous spectroscopic LRG surveys in areal coverage, redshift range, and overall number of targets.

\footnotetext{
3 http://www.legacysurvey.org/dr8/catalogs/\#galactic-extinction-coefficients

${ }^{4}$ http://www.legacysurvey.org/dr8/bitmasks/

${ }^{5}$ https://cmb-s4.org/wiki/images/DESI_L123_driver.pdf
} 

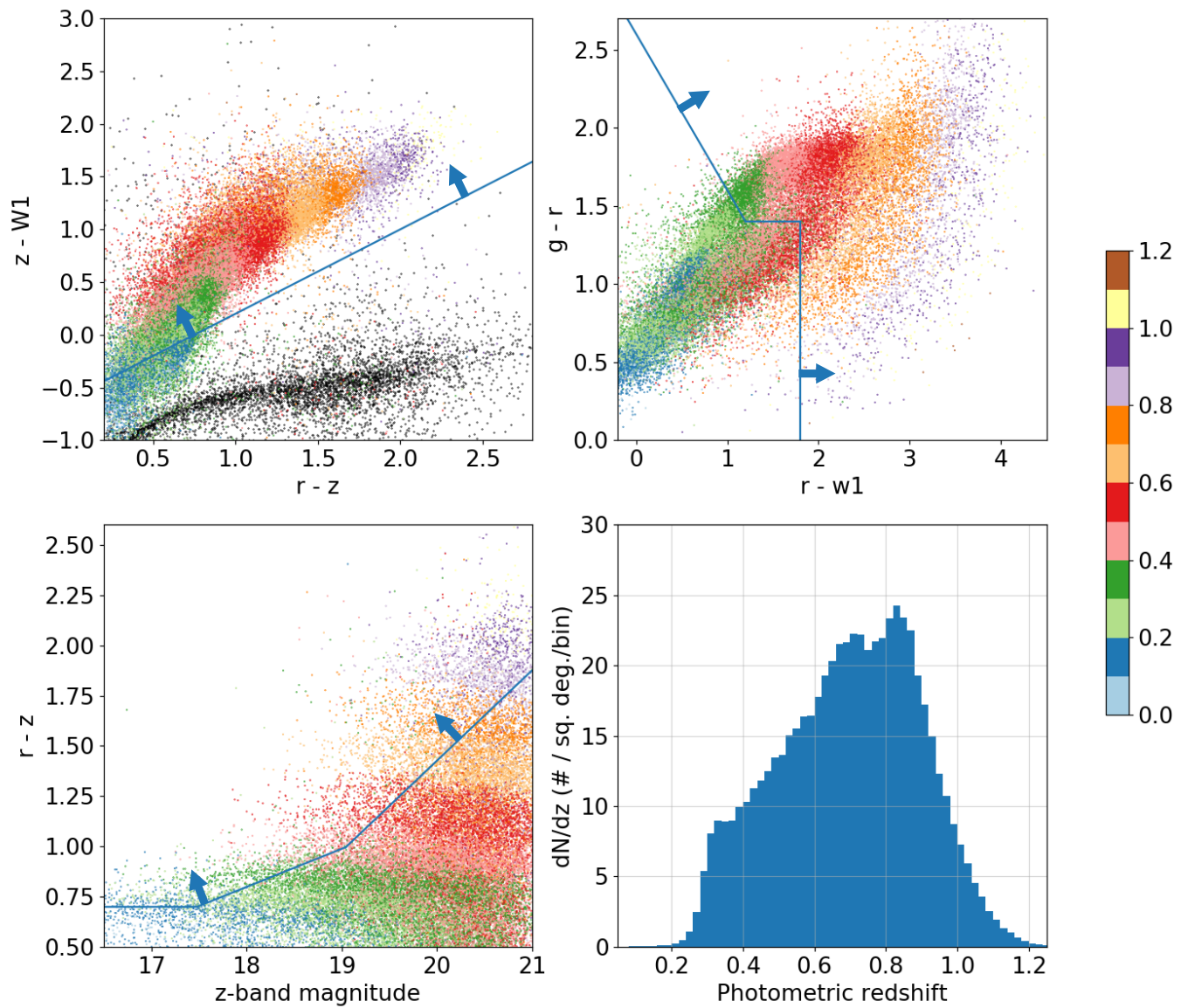

Figure 1. Illustration of the selection cuts for the DESI LRG sample, as listed in Equation 1. The first three panels present optical/near-infrared color-color and color-magnitude diagrams for objects from LS DR8. All extended sources with $z$-band fiber magnitude brighter than 21.5 are color-coded according to their PRLS photometric redshifts (Zhou et al. 2020). Blue lines depict the LRG selection boundaries given in Equation 1. In the upper left panel, we also plot the color-color distribution of point sources (mostly stars) in black. The lower right panel shows the photometric redshift distribution for the selected targets, showing the number of LRGs per $\operatorname{deg}^{2}$ in each $\Delta z=0.02$ redshift bin.

\section{ACKNOWLEDGEMENTS}

This research is supported by the Director, Office of Science, Office of High Energy Physics of the U.S. Department of Energy under Contract No. DE-AC02-05CH1123, and by the National Energy Research Scientific Computing Center, a DOE Office of Science User Facility under the same contract, as well as by an Office of High Energy Physics grant to the University of Pittsburgh. Additional support for DESI is provided by the U.S. National Science Foundation, Division of Astronomical Sciences under Contract No. AST-0950945 to the NSF's National Optical-Infrared Astronomy Research Laboratory; the Science and Technologies Facilities Council of the United Kingdom; the Gordon and Betty Moore Foundation; the Heising-Simons Foundation; the French Alternative Energies and Atomic Energy Commission (CEA); the National Council of Science and Technology of Mexico; the Ministry of Economy of Spain, and by the DESI Member Institutions. The authors are honored to be permitted to conduct astronomical research on Iolkam Du'ag (Kitt Peak), a mountain with particular significance to the Tohono O'odham Nation.

\section{REFERENCES}

Dawson, K. S., Kneib, J.-P., Percival, W. J., et al. 2016,

The Astronomical Journal, 151, 44,

doi: 10.3847/0004-6256/151/2/44
DESI Collaboration, Aghamousa, A., Aguilar, J., et al. 2016, arXiv e-prints, arXiv:1611.00036.

https://arxiv.org/abs/1611.00036 
Dey, A., Schlegel, D. J., Lang, D., et al. 2019, AJ, 157, 168, doi: 10.3847/1538-3881/ab089d

Eisenstein, D. J., Annis, J., Gunn, J. E., et al. 2001, The Astronomical Journal, 122, 2267, doi: 10.1086/323717

John, T. L. 1988, A\&A, 193, 189

Prakash, A., Licquia, T. C., Newman, J. A., \& Rao, S. M. 2015, The Astrophysical Journal, 803, 105, doi: 10.1088/0004-637X/803/2/105
Prakash, A., Licquia, T. C., Newman, J. A., et al. 2016, The Astrophysical Journal Supplement Series, 224, 34, doi: 10.3847/0067-0049/224/2/34

Reid, B., Ho, S., Padmanabhan, N., et al. 2016, Monthly Notices of the Royal Astronomical Society, 455, 1553, doi: 10.1093/mnras/stv2382

Sawicki, M. 2002, AJ, 124, 3050, doi: 10.1086/344682

Wright, E. L., Eisenhardt, P. R. M., Mainzer, A. K., et al. 2010, The Astronomical Journal, 140, 1868, doi: 10.1088/0004-6256/140/6/1868

Zhou, R., Newman, J. A., Mao, Y.-Y., et al. 2020, arXiv:2001.06018 [astro-ph].

https://arxiv.org/abs/2001.06018 